\title{
Pharmacy Technician Management of Stable, In-Range INRs Within a Clinical Pharmacy Anticoagulation Service
}

\author{
Kelsey L. Hawkins, PharmD; Jordan King, PharmD, MS; Thomas Delate, PhD, MS; \\ Kerri Martinez, PharmD; Kathleen McCool, PharmD; and Nathan P. Clark, PharmD
}

\begin{abstract}
BACKGROUND: There is increasing demand on pharmacist time within clinical pharmacy services, and pharmacy technicians are a crucial resource for expanding pharmacy practice.
\end{abstract}

OBJECTIVE: To assess the safety and effectiveness of pharmacy technician management of stable, in-range international normalized ratio (INR) results compared with usual care.

METHODS: This retrospective, longitudinal, noninferiority cohort study was conducted at an integrated health care delivery system with a centralized anticoagulation service. Adult patients receiving chronic warfarin therapy with therapeutic INR results over a 3-month period (i.e., 100\% time in therapeutic range [TTR] during the 3 months before the index date) were eligible for referral to technician warfarin management between March 1, 2015, and December 31, 2015. Patients with similar INR control during the same period but not referred to technician management were included as comparators in the usual care group. A one-sided noninferiority margin for the technician management group was set to $-2.5 \%$ for mean TTR. Propensity scoring was used in regression modeling via inverse probability of treatment weights to compare between-group differences to account for covariates that may have influenced assignment to the technician group. Finally, bleeding, thromboembolic, and mortality outcomes were compared.

RESULTS: 1,840 and 1,116 patients were included in the technician and usual care groups, respectively. The mean age of included patients was 73.1 years, and the majority $(77.9 \%)$ had received warfarin for $>3$ years. TTR during follow-up was $83.3 \%$ and $77.7 \%$ in the technician and usual care groups, respectively (mean difference $=5.7 \% ; 95 \% \mathrm{Cl}=4.1 \%-7.2 \%$ ). The risk of thromboembolism was similar between the technician and usual care groups ( $\mathrm{HR}=0.84 ; 95 \% \mathrm{Cl}=0.17-4.22 ; P=0.832$ ); however, bleeding $(\mathrm{HR}=0.60 ; 95 \% \mathrm{Cl}=0.39-0.94 ; P=0.026)$ and all-cause mortality ( $\mathrm{HR}=0.44 ; 95 \% \mathrm{Cl}=0.25-0.77 ; P=0.004$ ) were lower in the technician group during follow-up.

CONCLUSIONS: Technician management of stable patients receiving chronic warfarin therapy within an integrated health care delivery system's centralized anticoagulation service was associated with noninferior TTR results compared with usual care pharmacist management.

J Manag Care Spec Pharm. 2018;24(11):1130-37

Copyright @2018, Academy of Managed Care Pharmacy. All rights reserved.

\section{What is already known about this subject}

Expanded pharmacy technician duties have been implemented in several areas of clinical pharmacy, including medication reconciliation, chemotherapy order entry, and osteoporosis management. Several roles within anticoagulation clinics have been deemed appropriate for pharmacy technicians, such as triaging calls to pharmacists and reporting international normalized ratio [INR] values to patients.

\section{What this study adds}

It is feasible to expand pharmacy technician roles to include management of stable, in-range INR results in warfarin patients within an integrated health care delivery system's centralized anticoagulation service.

Time in therapeutic range among patients managed by pharmacy technicians was noninferior to patients managed by pharmacists. Implementation of technician management of stable, in-range INRs was not associated with an increased risk of bleeding compared with pharmacist management.

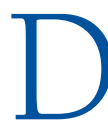
eveloping new clinical pharmacy interventions or expanding existing practice areas often require creating capacity with existing resources. ${ }^{1}$ Pharmacy technicians are a crucial resource in expanding pharmacy practice, and leveraging technicians can allow pharmacists to expand clinical services and focus time on patients requiring a higher level of care. There are several examples of expanded technician roles outside of a traditional pharmacy setting including medication reconciliation, chemotherapy order entry, postfracture osteoporosis management, and pharmacy data analysis. ${ }^{2-8}$ Although technicians can work effectively and safely in expanded clinical roles, there has been little research on technician involvement within anticoagulation clinics.

Pharmacy technicians have historically been involved in many operational and clerical roles within the anticoagulation clinic, including triaging phone calls, managing new patient intake, faxing laboratory results, entering laboratory tests into electronic medical records, and reporting international normalized ratio (INR) values to patients. Management of stable, in-range INRs by technicians is also likely appropriate, but there are limited data describing the safety or effectiveness of pharmacy technicians performing this duty. ${ }^{9}$

In 2015, the Clinical Pharmacy Anticoagulation and Anemia Service (CPAAS) at Kaiser Permanente Colorado (KPCO) developed a program whereby stable warfarin-receiving patients could be referred to pharmacy technician management. Within the protocol, future in-range INRs drawn on time and in the absence of new drug interactions could be managed by technicians without pharmacist review. The protocol was developed with the goals of expanding pharmacy technician roles to 


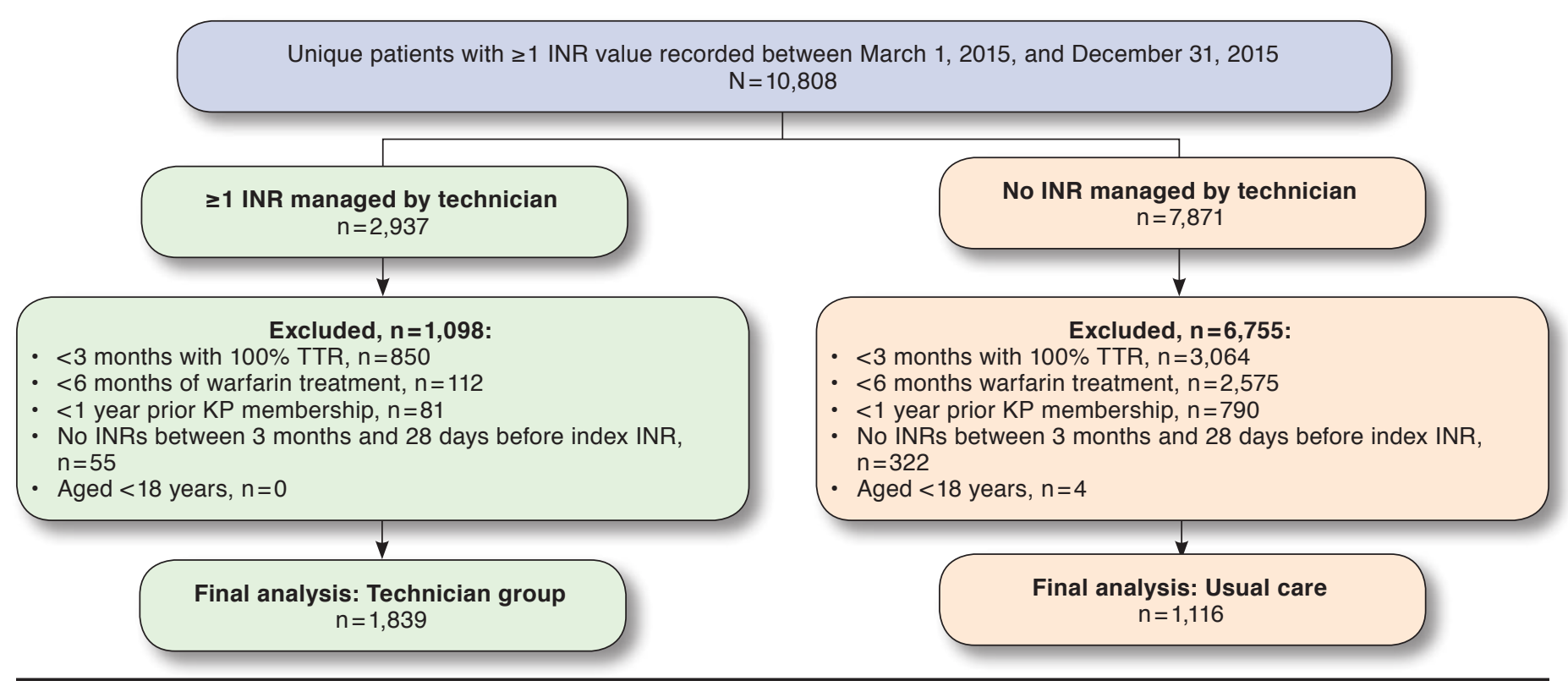

INR=international normalized ratio; $K P=$ Kaiser Permenante; TTR $=$ time in therapeutic range.

the top of their scope, increasing technician engagement, and creating capacity for clinical pharmacists at CPAAS.

The purpose of this retrospective, longitudinal, noninferiority study was to compare anticoagulation quality and safety outcomes between stable warfarin patients managed by a pharmacy technician protocol with similarly stable patients managed by usual care (i.e., clinical pharmacist management) to evaluate the effect of the technician protocol.

\section{Methods}

\section{Study Design, Setting, and Participants}

The KPCO program is an integrated health care delivery system providing comprehensive medical care to $>650,000$ members in the Denver/Boulder metropolitan area at 30 medical offices. CPAAS is a centralized pharmacy team that delivers comprehensive anticoagulation services for $>10,000$ KPCO members. ${ }^{10}$ The CPAAS team is made up of clinical pharmacists and certified pharmacy technicians. Pharmacy technicians receive on-the-job training regarding new policies and procedures, and their work is spot-checked by pharmacy supervisors at regular intervals.

Documentation of anticoagulation interventions and laboratory results is collated in DAWN-AC, a centralized electronic database (4S Systems, Cumbria, United Kingdom) that communicates with KPCO laboratory and health record systems via electronic interface. New INR results are transmitted from the KPCO laboratory information system into DAWN-AC where they are matched to an active patient according to name, date of birth, and medical record number. DAWN-AC automatically interprets the INR as in range or out of range and provides provisional warfarin dose and recheck INR recommendations per a computerized nomogram. The user can then either confirm or modify the provisional recommendation before accepting and finalizing the DAWN-AC treatment plan. Once the treatment plan is accepted, the warfarin dose, recommended recheck INR date, and method of communication to the patient are transmitted back to the laboratory information system and electronic medical record (EPIC, Verona, WI). Warfarin dose details are maintained within tables in DAWN-AC, which eliminates the need to retype or copy/paste dose information manually, thus reducing the potential for errors. Coded and free-text medical, pharmacy, laboratory, emergency department (ED), hospitalization, and membership information are captured in KPCO's administrative and claims databases.

The study population consisted of patients aged $\geq 18$ years receiving chronic anticoagulation with warfarin under CPAAS management for at least 6 months and receiving a stable warfarin dose. Patients were categorized to the technician group if they had at least 1 INR managed by a pharmacy technician between March 1, 2015, and December 31, 2015. All other patients were categorized into the usual care group. For patients referred to technician management, the index date was defined as the date of the first INR managed by a technician. For patients in the usual care group, the index date 
TABLE 1 Baseline Characteristics Before and After Inverse Probability of Treatment Weighting

\begin{tabular}{|c|c|c|c|c|c|c|c|}
\hline \multirow[b]{2}{*}{ Variable } & \multicolumn{4}{|c|}{ Before Weighting } & \multicolumn{3}{|c|}{ After Weighting } \\
\hline & $\begin{array}{c}\text { All Patients } \\
(\mathrm{N}=2,956)\end{array}$ & $\begin{array}{l}\text { Technician } \\
(\mathrm{n}=1,840)\end{array}$ & $\begin{array}{c}\text { Usual Care } \\
(\mathrm{n}=1,116)\end{array}$ & $\begin{array}{c}\text { Absolute } \\
\text { Standardized } \\
\text { Difference }\end{array}$ & $\begin{array}{l}\text { Technician } \\
\left(\mathrm{n}=1,839^{\mathrm{a}}\right)\end{array}$ & $\begin{array}{c}\text { Usual Care } \\
(\mathrm{n}=1,116)\end{array}$ & $\begin{array}{c}\text { Absolute } \\
\text { Standardized } \\
\text { Difference }\end{array}$ \\
\hline \multicolumn{8}{|l|}{ Demographics } \\
\hline Age, years, $\mathrm{n}( \pm \mathrm{SD})$ & $73.1 \pm 11.9$ & $73.4 \pm 11.2$ & $72.6 \pm 13$ & 0.075 & $73.1 \pm 17.1$ & $73.1 \pm 20.6$ & 0.002 \\
\hline$<65, \mathrm{n}(\%)$ & $669(22.6)$ & $383(20.8)$ & $286(25.6)$ & 0.115 & $(22.5)$ & $(23.0)$ & 0.009 \\
\hline $65-74, \mathrm{n}(\%)$ & $816(27.6)$ & $528(28.7)$ & $288(25.8)$ & 0.065 & $(28.1)$ & $(26.8)$ & 0.020 \\
\hline$\geq 75, \mathrm{n}(\%)$ & $1,471(49.8)$ & $929(50.5)$ & $542(48.6)$ & 0.038 & $(49.4)$ & $(50.2)$ & 0.010 \\
\hline Male sex, $\mathrm{n}(\%)$ & $1,618(54.7)$ & $1,010(54.9)$ & $608(54.5)$ & 0.008 & $(54.9)$ & $(55.0)$ & 0.001 \\
\hline \multicolumn{8}{|l|}{ Race/ethnicity, n (\%) } \\
\hline Non-Hispanic white & $2,509(84.9)$ & $1,557(84.6)$ & $952(85.3)$ & 0.019 & $(85.1)$ & $(85.3)$ & 0.004 \\
\hline Non-Hispanic black & $75 \quad(2.5)$ & $45 \quad(2.4)$ & $30 \quad(2.7)$ & 0.015 & $(2.5)$ & $(2.5)$ & 0.001 \\
\hline Hispanic & $188 \quad(6.4)$ & $114 \quad(6.2)$ & $74 \quad(6.6)$ & 0.018 & $(6.3)$ & $(6.2)$ & 0.003 \\
\hline Other & $96 \quad(3.2)$ & $72 \quad(3.9)$ & $24 \quad(2.2)$ & 0.100 & $(3.6)$ & $(2.4)$ & 0.050 \\
\hline \multicolumn{8}{|l|}{ Tobacco use $\mathrm{e}^{\mathrm{b}}$} \\
\hline Never, n (\%) & $1,421(48.1)$ & $861(46.8)$ & $560(50.2)$ & 0.068 & $(48.1)$ & $(48.4)$ & 0.005 \\
\hline Quit, n (\%) & $1,345(45.5)$ & $864(47)$ & $481(43.1)$ & 0.077 & $(45.4)$ & $(45.5)$ & 0.001 \\
\hline Current, n (\%) & $173 \quad(5.9)$ & $102 \quad(5.5)$ & $71 \quad(6.4)$ & 0.035 & $(5.8)$ & $(5.8)$ & 0.000 \\
\hline $\mathrm{BMI}^{\mathrm{b}}, \mathrm{n}( \pm \mathrm{SD})$ & $30 \pm 11.5$ & $29.9 \pm 6.2$ & $30.1 \pm 17$ & 0.019 & $29.9 \pm 15.4$ & $29.9 \pm 22.9$ & 0.001 \\
\hline \multicolumn{8}{|c|}{ Duration of warfarin treatment episode,,$^{\mathrm{c}} \mathbf{n}(\%)$} \\
\hline$<1$ year & $225(7.6)$ & $124 \quad(6.7)$ & $101 \quad(9.1 \%)$ & 0.087 & $(7.5)$ & $(7.4)$ & 0.001 \\
\hline $2-3$ years & $429(14.5)$ & $271(14.7)$ & $158(14.2)$ & 0.016 & $(14.5)$ & $(14.7)$ & 0.004 \\
\hline$\geq 3$ years & $2,302(77.9)$ & $1,445(78.5)$ & $857(76.8)$ & 0.042 & $(78.0)$ & $(77.8)$ & 0.002 \\
\hline \multicolumn{8}{|l|}{ Warfarin indication, $\mathrm{n}(\%)$} \\
\hline Mechanical heart valve & $186 \quad(6.3)$ & $102 \quad(5.5)$ & $84 \quad(7.5)$ & 0.082 & $(6.4)$ & $(6.4)$ & 0.001 \\
\hline Atrial fibrillation & $1,682(56.9)$ & $1,080(58.7)$ & $602(53.9)$ & 0.096 & $(56.9)$ & $(56.9)$ & 0.001 \\
\hline Venous thromboembolism & $930(31.5)$ & $568(30.9)$ & $362(32.4)$ & 0.034 & $(31.3)$ & $(31.4)$ & 0.000 \\
\hline Other & $157 \quad(5.3)$ & $89 \quad(4.8)$ & $68 \quad(6.1)$ & 0.056 & $(5.3)$ & $(5.4)$ & 0.003 \\
\hline \multicolumn{8}{|l|}{ INR goal, ${ }^{b}$ n (\%) } \\
\hline $2.0-3.0$ & $2,825(95.6)$ & $1,777(96.6)$ & $1,048(93.9)$ & 0.130 & $(96.0)$ & $(94.5)$ & 0.049 \\
\hline $2.5-3.5$ & $131 \quad(4.4)$ & $63 \quad(3.4)$ & $68 \quad(6.1)$ & 0.130 & $(4.0)$ & $(5.5)$ & 0.049 \\
\hline \multicolumn{8}{|l|}{ Prior bleeding, ${ }^{\mathrm{b}}$ n (\%) } \\
\hline Hemorrhagic stroke & $11 \quad(0.4)$ & $6 \quad(0.3)$ & $5 \quad(0.4)$ & 0.020 & $(0.4)$ & $(0.4)$ & 0.001 \\
\hline GI bleeding & $48 \quad(1.6)$ & $22 \quad(1.2)$ & $26 \quad(2.3)$ & 0.090 & $(1.7)$ & $(1.7)$ & 0.000 \\
\hline Other bleeding & $274 \quad(9.3)$ & $164 \quad(8.9)$ & $110 \quad(9.9)$ & 0.033 & $(9.3)$ & $(9.3)$ & 0.001 \\
\hline
\end{tabular}

was defined as the first INR on or after March 1, 2015. Stable warfarin therapy was defined as (a) no change in the INR goal for at least 6 months immediately preceding the index date, (b) all INR values in the therapeutic range in the 90 days immediately preceding the index date, and (c) at least 1 INR occurring $>28$ days before the index date. Patients were excluded from analysis if they had $<12$ months of KPCO membership before the index date, no available follow-up after the index date, or no documented INR goal listed in DAWN-AC. Patients were censored from the study upon KPCO membership termination, death, a safety event (bleeding or thromboembolic event), or at 6 months, whichever occurred first. Once patients were categorized to the technician management group, they remained within the technician group throughout the study follow-up period, even if care was later transitioned back to usual care (e.g., for an out-of-range INR or a new drug interaction). All study activities were reviewed and approved by the KPCO Institutional Review Board.

\section{Intervention}

Within the technician management group, in-range INR results were managed by pharmacy technicians without pharmacist oversight. If an INR result was in range and performed on time, the managing technician notified the patient to continue their current warfarin dose via letter or secure email. Patients with out-of-range INRs, newly initiated medications with the potential for warfarin interactions identified through automated daily pharmacy reports, nonadherence to INR testing, or unexpected early INR results were transitioned back to their clinical pharmacist for follow-up. Pharmacy technicians were not permitted to make warfarin dose changes, interpret 
TABLE 1 Baseline Characteristics Before and After Inverse Probability of Treatment Weighting (continued)

\begin{tabular}{|c|c|c|c|c|c|c|c|}
\hline \multirow[b]{2}{*}{ Variable } & \multicolumn{4}{|c|}{ Before Weighting } & \multicolumn{3}{|c|}{ After Weighting } \\
\hline & $\begin{array}{l}\text { All Patients } \\
(\mathrm{N}=2,956)\end{array}$ & $\begin{array}{l}\text { Technician } \\
(\mathrm{n}=1,840)\end{array}$ & $\begin{array}{l}\text { Usual Care } \\
(\mathrm{n}=1,116)\end{array}$ & $\begin{array}{c}\text { Absolute } \\
\text { Standardized } \\
\text { Difference }\end{array}$ & $\begin{array}{l}\text { Technician } \\
\left(\mathrm{n}=1,839^{\mathrm{a}}\right)\end{array}$ & $\begin{array}{l}\text { Usual Care } \\
(\mathrm{n}=1,116)\end{array}$ & $\begin{array}{c}\text { Absolute } \\
\text { Standardized } \\
\text { Difference }\end{array}$ \\
\hline \multicolumn{8}{|c|}{ Other past medical history, ${ }^{b}$ n (\%) } \\
\hline Heart failure & $641(21.7)$ & $395(21.5)$ & $246(22)$ & 0.014 & $(21.7)$ & $(21.6)$ & 0.000 \\
\hline Diabetes mellitus & $788(26.7)$ & $483(26.3)$ & $305(27.3)$ & 0.024 & $(26.7)$ & $(26.7)$ & 0.000 \\
\hline Hypertension & $1,760(59.5)$ & $1,098(59.7)$ & $662(59.3)$ & 0.007 & $(59.4)$ & $(59.4)$ & 0.001 \\
\hline CAD with history of MI & $281 \quad(9.5)$ & $163 \quad(8.9)$ & $118(10.6)$ & 0.058 & $(9.5)$ & $(9.5)$ & 0.000 \\
\hline CAD without history of MI & $299(10.1)$ & $183 \quad(9.9)$ & $116(10.4)$ & 0.015 & $(10.1)$ & $(10.1)$ & 0.000 \\
\hline History of ischemic stroke/TIA & $494(16.7)$ & $288(15.7)$ & $206(18.5)$ & 0.051 & $(17.1)$ & $(17.2)$ & 0.001 \\
\hline Chronic kidney disease & $1,022(34.6)$ & $653(35.5)$ & $369(33.1)$ & 0.051 & $(34.6)$ & $(34.8)$ & 0.002 \\
\hline Liver dysfunction & $63 \quad(2.1)$ & $39(2.1)$ & $24(2.2)$ & 0.002 & $(2.1)$ & $(2.1)$ & 0.001 \\
\hline \multicolumn{8}{|c|}{ Recent health care utilization, ${ }^{\mathrm{b}} \mathrm{n}(\%)$} \\
\hline Inpatient admission & $361(12.2)$ & $196(10.7)$ & $165(14.8)$ & 0.126 & $(12.1)$ & $(12.0)$ & 0.001 \\
\hline ED visit & $700(23.7)$ & $390(21.2)$ & $310(27.8)$ & 0.155 & $(23.7)$ & (23.8) & 0.001 \\
\hline \multicolumn{8}{|l|}{ Concurrent medications, $\mathrm{n}(\%)$} \\
\hline Clopidogrel & $32(1.1)$ & $14 \quad(0.8)$ & $18 \quad(1.6)$ & 0.082 & $(1.0)$ & $(1.1)$ & 0.001 \\
\hline Aspirin & $377(12.8)$ & $218(11.8)$ & $159(14.2)$ & 0.072 & $(12.8)$ & $(12.7)$ & 0.002 \\
\hline \multicolumn{8}{|l|}{ Risk scores, n (\%) } \\
\hline \multicolumn{8}{|l|}{$\mathrm{CHA}_{2} \mathrm{DS}_{2}-\mathrm{VASC}^{\mathrm{d}}$} \\
\hline 0 & $201 \quad(6.8)$ & $117 \quad(6.4)$ & $84 \quad(7.5)$ & 0.046 & $(6.7)$ & $(7.0)$ & 0.010 \\
\hline $1-2$ & $851(28.8)$ & $531(28.9)$ & $320(28.7)$ & 0.004 & $(29.0)$ & $(28.6)$ & 0.005 \\
\hline 3 & $1,904(64.4)$ & $1,192(64.8)$ & $712(63.8)$ & 0.021 & $(64.4)$ & $(64.4)$ & 0.000 \\
\hline \multicolumn{8}{|l|}{ HAS-BLEDe } \\
\hline 0 & $347(11.7)$ & $202(11)$ & $145(13)$ & 0.063 & $(11.6)$ & $(11.8)$ & 0.005 \\
\hline $1-2$ & $1,579(53.4)$ & $989(53.8)$ & $590(52.9)$ & 0.018 & $(53.4)$ & $(53.9)$ & 0.008 \\
\hline 3 & $1,030(34.8)$ & $649(35.3)$ & $381(34.1)$ & 0.024 & $(35.0)$ & (34.3) & 0.011 \\
\hline
\end{tabular}

Note: Age was calculated as of the index date.

${ }^{a}$ One of the 1,840 patients in the technician group returned a missing value when calculating the inverse probability of treatment weights.

${ }^{b}$ In 180 days before index date.

'Based on DAWN-AC treatment plan.

${ }^{d} \mathrm{CHA}_{2} \mathrm{DS}_{2}$-VASc score = congestive heart failure, hypertension, age $\geq 75$ years (2 points), diabetes mellitus, stroke (2 points), vascular disease, age 65-74 years, and sex (female).

${ }^{e}$ HAS-BLED score = hypertension, abnormal renal/liver function, stroke, bleeding history or predisposition, labile INR, elderly (>65 years), drugs/alcohol concomitantly, for prediction of bleeding risk.

$B M I=$ body mass index; $C A D=$ coronary artery disease; $E D=$ emergency department; $I N R=$ international normalized ratio; $M I=$ myocardial infarction; $S D=s t a n d a r d$ deviation; TIA = transient ischemic attack.

drug interactions, or adjust the provisional dose and recheck INR interval provided by DAWN-AC. Patients included in the usual care group were managed solely by clinical pharmacists and did not receive care from a pharmacy technician.

\section{Study Outcomes}

The primary outcome was 6-month time in therapeutic range (TTR). All INR values in DAWN-AC in the 6 months after the index date were used to calculate TTR. We used the method of linear interpolation as described by Rosendaal et al. (1993) for this calculation. ${ }^{11}$ Patients were required to have at least 2 INRs in the post-index period, and we allowed for gaps of up to 150 days between INRs in order to calculate TTR. Secondary outcomes included 6-month rates of bleeding events, thrombotic events, and all-cause mortality. Potential bleeding and thrombotic events were identified via ED or hospitalization discharge International Classification of Diseases, Ninth/Tenth Revision, Clinical Modification (ICD-9/10-CM) codes (Appendix A, available in online article) and adjudicated with manual chart review. ${ }^{12}$ Clinically relevant bleeding events were defined as any overt bleeding that led to a hospitalization or ED visit. Confirmed thromboembolic events also required a hospitalization and/or ED visit with appropriate objective diagnostic confirmation (e.g., evidence of thrombus on computed tomography or Doppler ultrasonography).

\section{Data Collection}

Information including patient age, sex, health plan membership dates, race/ethnicity, and death date was obtained from queries of the KPCO electronic membership database. INR 


TABLE 2 INR Control During Follow-up by
Warfarin Management Group

measurement dates and values were obtained from the KPCO electronic laboratory database. Medication dispensing data were obtained from queries of the KPCO electronic pharmacy dispensing database. Additional demographic information including comorbidities, body mass index, and tobacco use was obtained from the KPCO electronic clinic visits database using predefined ICD-9-CM and ICD-10-CM codes (Appendix B, available in online article). Information regarding the primary indication for anticoagulation, warfarin dose, and invasive procedures was obtained from queries of the DAWN-AC database.

\section{Data Analysis}

The primary analysis was designed to test whether technician management was noninferior to usual care, as determined by 6 -month TTR. There is no established standard for the acceptable margin in noninferiority comparisons of TTR. Earlier studies have used noninferiority margins of $-4.5 \%$ and $-7.5 \%$ for change in mean TTR. ${ }^{13,14}$ We felt these thresholds were too liberal and elected to set a noninferiority margin of $-2.5 \%$ a priori for analysis of the primary outcome. This margin hypothesizes that the mean TTR in the technician group would be no lower than $2.5 \%$ below the usual care group mean TTR. All analyses were based on the intention-to-treat principle.

Propensity score analysis was used to identify and adjust for variables that may have accounted for a patient being assigned to the technician group rather than usual care..$^{15}$ The propensity score was calculated from a logistic regression model with the technician group as the dependent variable and patient or clinical characteristics as independent variables. Patient demographic characteristics were extracted from KPCO mem- bership files, and comorbidities and concomitant medications were determined from the presence or absence of diagnosis and pharmacy records, respectively, in the year immediately preceding the index date. Diagnosis codes used are provided in the appendices. Patient characteristics in this study were determined a priori based on clinical judgment and included age; patient sex; race and ethnicity; body mass index; tobacco use; history of alcohol abuse; indication for anticoagulation; warfarin target INR range; the number of INRs obtained in the pre-index period; prior history of thromboembolic event; prior history of gastrointestinal bleed, hemorrhagic stroke, or other bleed; heart failure; diabetes mellitus; hypertension; history of myocardial infarction; coronary artery disease without a history of myocardial infarction; renal dysfunction; liver dysfunction; peripheral artery disease or aortic plaque; recent hospitalization or ED health encounters; concomitant use of clopidogrel, aspirin, nonsteroidal anti-inflammatory drugs, estrogen, amiodarone, dronedarone, and corticosteroids; $\mathrm{CHA}_{2} \mathrm{DS}_{2}$-VASc risk score; and HAS-BLED risk score. ${ }^{16,17}$ The propensity score for each patient was the predicted probability calculated from the final model. ${ }^{15}$ The propensity score was implemented during regression analysis via inverse probability of treatment weights, and differences in baseline characteristics between groups were assigned before and after weighting using standardized mean differences. A difference of $<0.1$ was considered nonclinically meaningful.

Linear regression models were used to compare the TTR between groups, and logistic regression was used to compare the of probability of achieving or not achieving 100\% TTR during follow-up. Cox proportional hazards models were used to estimate hazard ratios (HRs) and their 95\% confidence intervals (CIs) associating technician versus usual care (independent variable) with thromboembolic, bleeding, and mortality outcomes (dependent variables). A separate Cox model was developed for each outcome, as well as a model for the composite of all 3. All models, including the linear regression and the Cox proportional hazards regression, were weighted using inverse probability of treatment weights. Data analysis was performed using Stata/SE Version 14.1 (StataCorp, College Station, TX) and SAS 9.4 statistical software (SAS Institute, Cary, NC).

\section{Results}

A total of 1,840 patients and 1,116 patients were included in the technician and usual care groups, respectively (Figure 1). After propensity weighting, the groups were well balanced with respect to age, sex, race, duration of warfarin use, indication for warfarin use, INR target goal, $\mathrm{CHA}_{2} \mathrm{DS}_{2}$-VASc score, and HAS-BLED score (Table 1). Median follow-up was 5.9 months, and $44(2.4 \%)$ patients in the technician group and 42 (3.8\%) patients in the usual care group were censored before 6 months due to termination of KPCO membership. 


\section{FIGURE 2 Mean Time in Therapeutic Range According to INR Management Group}

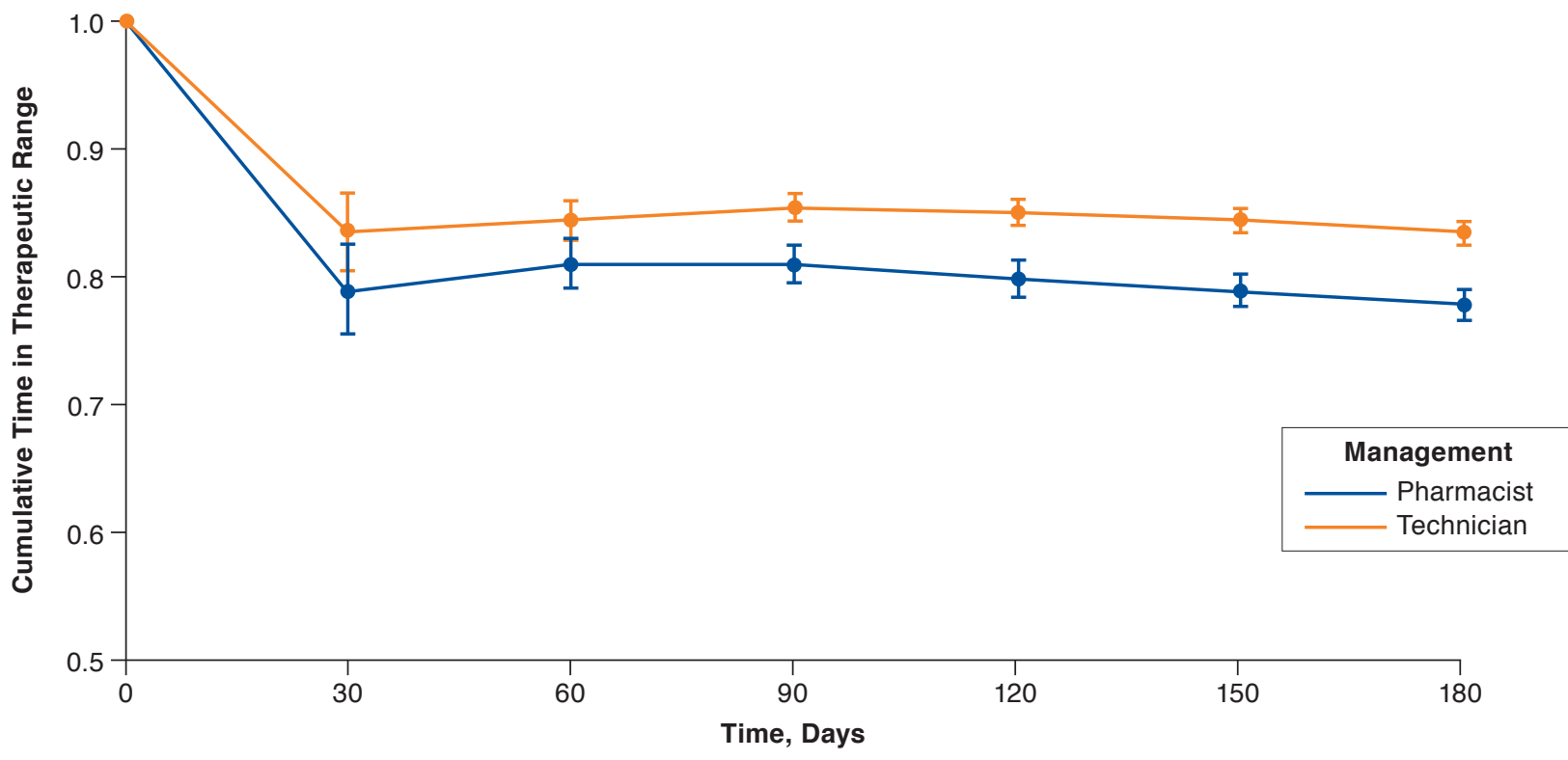

Note: Error bars around point estimates are 95\% CIs. Technician INR management was noninferior to pharmacist INR management at 180 days (mean adjusted TTR increased $5.7 \%$; $95 \%$ CI $4.1 \%-7.2 \%$ ).

$C I=$ confidence interval; INR=international normalized ratio; TTR= time in therapeutic range.

The propensity-weighted 6-month follow-up mean TTR was $83.3 \%(95 \% \mathrm{CI}=82.4 \%-84.2 \%)$ in the technician group and $77.7 \%(95 \% \mathrm{CI}=76.4 \%-78.9 \%)$ in the usual care group, with a mean difference of $5.7 \%$ (95\% CI=4.1\%-7.2\%; Table 2). The mean difference did not cross the noninferiority margin of $-2.5 \%$, indicating that technician management was noninferior to usual care management (Figure 2). The technician group had a lower mean number of INRs measured (mean difference $=-0.6 ; 95 \% \mathrm{CI}=-0.9,-0.4$; Table 2). Additionally, the technician group had a higher percentage of in-range INRs (mean difference $=6.8 \% ; 95 \% \mathrm{CI}=5.0 \%-8.7 \%)$ and patients with $100 \%$ TTR (mean difference $=10.5 \%$; 95\% CI $=7.0 \%-14.0 \%$ ) during follow-up.

Bleeding and thromboembolic outcomes were rare in both groups (Table 3). There were 3 thromboembolic events in each of the technician and usual care groups, corresponding to rates of 0.5 and 0.6 events per 100 patient-years, respectively ( $\mathrm{HR}=0.84 ; 95 \% \mathrm{CI}=0.17-4.22 ; P=0.832$ ). Clinically relevant bleeding occurred in 41 and 40 patients in the technician and usual care groups, corresponding to rates of 4.4 and 7.3 events per 100 patient-years $(\mathrm{HR}=0.60 ; 95 \% \mathrm{CI}=0.39-0.77$; $P=0.026$ ). Death from any cause occurred in 22 and 29 patients in the technician and usual care groups, respectively (HR $=0.44 ; 95 \% \mathrm{CI}=0.25-0.77 ; P=0.004)$.

\section{Discussion}

This study examined warfarin therapy quality measures after implementation of a protocol permitting pharmacy technician management of stable, in-range INRs in the setting of an integrated delivery system centralized pharmacy anticoagulation service. In our propensity-weighted analysis, we found no evidence of loss of INR control, since the 6-month follow-up technician group TTR did not cross the noninferiority margin of $-2.5 \%$. In addition, we identified no evidence that technician management resulted in excess thromboembolic or clinically relevant bleeding outcomes. Our findings suggest that pharmacy technician management of stable, in-range INRs is feasible within a centralized pharmacy anticoagulation service without compromising high-quality anticoagulation care.

We identified that both the technician and usual care groups had high 6-month TTR ( $>75 \%$ ) and low rates of thromboembolic and bleeding events. Comparing these outcomes to those reported in other warfarin populations would not be valid as we designed this study to specifically include stable warfarin patients only. While the technician group had higher TTR and lower bleeding and mortality outcomes than the usual care group during follow-up, it is unclear if this may have been due to unmeasured factors that resulted in unbalanced risk at baseline that we were unable to account for using the propensity score adjustment. Clinical pharmacists at CPAAS were 
TABLE 3 Clinical Outcomes During Follow-up

\begin{tabular}{|c|c|c|c|c|c|c|c|c|}
\hline \multirow[b]{3}{*}{ Variable } & \multicolumn{3}{|c|}{ Technician } & \multicolumn{3}{|c|}{ Pharmacist } & \multirow{3}{*}{$\begin{array}{c}\text { Hazard Ratio } \\
(95 \% \mathrm{CI})\end{array}$} & \multirow[b]{3}{*}{$P$ Value } \\
\hline & & $\%)$ & $\%$ per year & & $\%)$ & $\%$ per year & & \\
\hline & \multicolumn{3}{|c|}{$\left(n=1,839^{a}\right)$} & \multicolumn{3}{|c|}{$(n=1,116)$} & & \\
\hline Thromboembolism & 3 & $(0.2)$ & 0.5 & 3 & $(0.3)$ & 0.6 & $0.84 \quad(0.17-4.22)$ & 0.832 \\
\hline Bleeding & 41 & $(2.2)$ & 4.4 & 40 & (3.6) & 7.3 & $0.60(0.39-0.94)$ & 0.026 \\
\hline All-cause mortality & 22 & (1.2) & 2.3 & 29 & (2.6) & 5.4 & $0.44 \quad(0.25-0.77)$ & 0.004 \\
\hline Composite endpoint ${ }^{\mathrm{b}}$ & 62 & $(3.4)$ & 6.8 & 68 & $(6.1)$ & 12.6 & $0.54(0.38-0.77)$ & 0.001 \\
\hline \multicolumn{9}{|c|}{$\begin{array}{l}\text { Note: Number of patients (\%) are unadjusted values. \% per year rates, hazard ratios, } 95 \% \text { CIs, and P values were obtained from data adjusted using IPTW. } \\
\text { a One of the 1,840 patients in the technician group returned a missing value when calculating the inverse probability of treatment weights. } \\
\text { bComposite of thromboembolism, bleeding, and mortality. Fatal bleeding and thromboembolic events were counted only once. } \\
\text { CI= confidence interval; INR=international normalized ratio; IPTW=inverse probability of treatment weighting. }\end{array}$} \\
\hline
\end{tabular}

permitted to use their clinical judgment in deciding which stable patients could be transitioned to the technician panel. As a result, it is possible that they elected to keep patients with low health literacy, barriers to adherence, and/or terminal illness.

Advanced pharmacy technician roles have been associated with improved outcomes in other roles and disease states. For example, medication reconciliation by technicians was associated with reduced drug omissions, drug errors, and inappropriate prescribing compared with usual care..$^{3-5}$ Expanded technician roles in oncology found that chemotherapy order entry was as safe and efficient as pharmacist ordering in an outpatient oncology treatment center. ${ }^{6}$ Although a specific quality advantage was not identified, trading technician effort to save pharmacist time is cost saving. ${ }^{7}$ Similarly, a study evaluating pharmacy technician intervention in osteoporosis management found that technicians could assist in identifying eligible post-fracture patients and reliably collect relevant clinical information from the electronic medical record. ${ }^{8}$ Compared to usual care, technician involvement resulted in numerically less pharmacist time spent on the intervention among eligible patients $(13.5 \pm 7.1$ minutes vs. $18.2 \pm 16.6$ minutes; $P=0.34) .{ }^{8}$

A community hospital in Canada conducted a pilot program to assess the competency of clinical pharmacy support assistants in management of patients receiving warfarin therapy. ${ }^{18}$ The support assistants were trained by pharmacists during 2 didactic sessions after which the assistants were required to pass a quiz. Unlike our study, Man et al. (2014) tested whether assistants could select an appropriate warfarin dose according to a recent INR result. ${ }^{18}$ The study found that warfarin dosing recommendations by clinical pharmacy support assistants were similar to pharmacist recommendations, differing only by a mean $0.46 \mathrm{mg}$. In $65 \%$ of the patient encounters, the dose decisions for clinical pharmacists and the support assistants were identical. The maximum difference in any warfarin dose selection was $3 \mathrm{mg}$, which occurred just 1 time. Man et al. suggest that support staff can be trained to accurately select warfarin dose adjustments. However, the program did not permit pharmacy technicians to act on INR results without pharmacist review. It was not clear from the publication if pharmacist review was required only as a part of the research project or if review would always be required as a part of usual care. Board of pharmacy rules do not typically allow for clinical decision making to be delegated, so it is probable that any U.S. adaptation of this project would always require pharmacist review, which limits, to some extent, the amount of pharmacist time saved.

Our study adds to this growing body of literature that supports the expansion of pharmacy technician roles to include management of stable, in-range INRs, which may create capacity for clinical pharmacists to manage more complex patients or expand the breadth of services provided. Expanded technician roles have contributed importantly to the ability of CPAAS to continue to grow with existing clinical pharmacist resources. Since 2014, CPAAS has seen a 9\% increase in its warfarin population and has extended anticoagulation management activities to more than a thousand patients taking direct-acting oral anticoagulants. Strengths of the present study include a large sample size (i.e., > 1,000 patients in each group) and use of propensity score weighting to minimize the effect of baseline differences between groups on study outcomes. In addition, the setting of the study within an integrated health care delivery system minimizes the likelihood that lab results or clinical events were missed.

\section{Limitations}

There are several limitations to our study. The group assignment does not present a pure comparison of technician and pharmacist warfarin management, since any patient assigned to technician management with an out-of-range INR, new drug-drug interaction, or nonadherence was transitioned back to pharmacist management. Because patients with these clinical concerns were immediately referred back to management by a clinical pharmacist, analyzing patients according to actual management received (i.e., time-varying exposure) would induce bias due to technicians only managing in-range INRs. Propensity score adjustment was performed to mitigate the risk 
of allocation bias, which can only be completely overcome by comparing technician management to usual care in a randomized controlled trial.

We relied on administrative data for initial identification of bleeding and thromboembolic outcomes and, as such, it is possible that some events may have been missed if not appropriately coded. However, it is unlikely that miscoded events would have occurred disproportionately in the 2 study groups. In addition, the study was not sufficiently powered to rule out small differences in the risk of thromboembolism.

Finally, this study was conducted in a single anticoagulation service center within an integrated health care delivery system. The feasibility of technician management of stable, in-range INRs may differ in other settings.

\section{Conclusions}

Technician management of stable, in-range INRs within an integrated health care delivery system's centralized anticoagulation service was associated with noninferior TTR and safety outcomes compared with usual care. These results suggest that expansion of pharmacy technician duties within anticoagulation services to include in-range INR management is feasible. Further research is needed to explore involvement of pharmacy technicians in warfarin management in a wider array of health care settings.

\section{Authors}

KELSEY L. HAWKINS, PharmD, Kaiser Permanente Northwest, Clackamas, Oregon. JORDAN KING, PharmD, MS; THOMAS DELATE, PhD, MS; KERRI MARTINEZ, PharmD; KATHLEEN MCCOOL, PharmD; and NATHAN P. CLARK, PharmD, Kaiser Permanente Colorado, Aurora.

AUTHOR CORRESPONDENCE: Nathan Clark, PharmD, Kaiser Permanente Colorado, 16601 E. Centretech Pkwy., Aurora, CO 80011. Tel.: 303.739.4901; E-mail: Nathan.Clark@kp.org.

\section{DISCLOSURES}

This study was internally funded by the Kaiser Permanente Pharmacy Department. The study sponsor had no role in the study design, analysis, or interpretation. The authors have no relevant financial conflicts of interest to disclose.

\section{REFERENCES}

1. Pedersen CA, Schneider PJ, Scheckelhoff DJ. ASHP national survey of pharmacy practice in hospital settings: prescribing and transcribing. Am J Health Syst Pharm. 2014;71(11):924-42.
2. Cater SW, Luzum M, Serra AE, et al. A prospective cohort study of medication reconciliation using pharmacy technicians in the emergency department to reduce medication errors among admitted patients. J Emerg Med. 2015;48(2):230-38.

3. Van Der Bemt PM, Van Den Broek S, Van Nunen AK, Harbers JB Lenderink AW. Medication reconciliation performed by pharmacy technicians at the time of preoperative screening. Ann Pharmacother. 2009;43(5):868-74.

4. Sen S, Siemianowski L, Murphy M, McAllister SC. Implementation of a pharmacy technician-centered medication reconciliation program at an urban teaching medical center. Am J Health Syst Pharm. 2014;71(1):51-56.

5. Pavlov A, Muravyev R, Amoateng-Adjepong Y, Manthous CA. Inappropriate discharge on bronchodilators and acid-blocking medication after ICU admission: importance of medication reconciliation. Respir Care. 2014;59(10):1524-29.

6. Neville H, Broadfield L, Harding C, et al. Chemotherapy order entry by a clinical support pharmacy technician in an outpatient medical day unit. Can J Hosp Pharm. 2016;69(3):202-08.

7. Ervin KC, Skledar S, Hess MM, Ryan M. Data analyst technician: an innovative role for the pharmacy technician. Am J Health Syst Pharm. 2001;58(19):1815-18.

8. Irwin AN, Heilmann RM, Gerrity TM, Kroner BA, Olson KL. Use of pharmacy technician to facilitate postfracture care provided by clinical pharmacy specialists. Am J Health Syst Pharm. 2014;71(24):2054-59.

9. Kuhn H, Park A, Kim B, Lukesh W, Rose A. Proportion of work appropriate for pharmacy technicians in anticoagulation clinics. Am J Health Syst Pharm. 2016;73(5):322-27.

10. Witt DM, Sadler MA, Shanahan RL, Mazzoli G, Tillman DJ. Effect of a centralized clinical pharmacy anticoagulation service on the outcomes of anticoagulation therapy. Chest. 2005;127(5):1515-22.

11. Rosendaal FR, Cannegieter SC, van der Meer FJ, Briet E. A method to determine the optimal intensity of oral anticoagulant therapy. Thromb Haemost. 1993;69(3):236-39.

12. Quan H, Sundararajan V, Halfon P, et al. Coding algorithms for defining comorbidities in ICD-9-CM and ICD-10 administrative data. Med Care. 2005;43(11):1130-39.

13. Schulman S, Parpia S, Stewart C, et al. Warfarin dose assessment every 4 weeks versus every 12 weeks in patients with stable international normalized ratios: a randomized trial. Ann Intern Med. 2011;155(1):653-59.

14. Nieuwlaat R, Hubers LM, Spyropoulos AC, et al. Randomised comparison of a simple warfarin dosing algorithm versus a computerized anticoagulation management system for control of warfarin maintenance therapy. Thromb Haemost. 2012;108(6):1228-35.

15. D'Agostino RB Jr. Propensity score methods for bias reduction in the comparison of a treatment to a non-randomized control group. Stat Med. 1998;17(19):2265-81.

16. Lip GY, Nieuwlaat R, Pisters R, Lane DA, Crijns HJ. Refining clinical risk stratification for predicting stroke and thromboembolism in atrial fibrillation using a novel risk factor-based approach: the euro heart survey on atrial fibrillation. Chest. 2010;137(2):263-72.

17. Lip GY, Frison L, Halperin JL, Lane DA. Comparative validation of a novel risk score for predicting bleeding risk in anticoagulated patients with atrial fibrillation: the HAS-BLED (Hypertension, Abnormal Renal/Liver Function, Stroke, Bleeding History or Predisposition, Labile INR, Elderly, Drugs/Alcohol Concomitantly) score. J Am Coll Cardiol. 2011;57(2):173-80.

18. Man D, Mabasa VH. Feasibility of assisted warfarin dosing by clinical pharmacy support assistants. Can J Hosp Pharm. 2014;67(3):220-25. 


\section{APPENDIX A Emergency Department and Hospitalization Discharge Diagnosis Codes Used to Identify Potential Outcome Events}

\begin{tabular}{|c|c|}
\hline Outcome & Definition \\
\hline \multirow[t]{2}{*}{ Thromboembolisma } & $\begin{array}{l}\text { ICD-9-CM: 444.x, 325, 362.3x (except 362.37), 362.84, 435.8, 435.9, 436, 452, 453.0, 453.1, 453.2, 453.3, 453.8, 453.9, 459.1, } \\
\text { 557.0, 557.9, 996.71, 996.73, 415.x, 433.x, 434.x, 451.x, 671.x, 673.x }\end{array}$ \\
\hline & $\begin{array}{l}\text { ICD-10-CM: H34.1x, H34.2x, H34.81x, H34.9, H35.82, I26.02, I26.09, I26.92, I26.99, I63.x, I67.82, I74.x, N28.0, I80.x (except } \\
\text { I80.0x), I81, I82.0, I82.1, I82.210, I82.220, I82.290, I82.3, I82.4x, I82.60x, I82.62x, I82.890, I82.90, I82.Alx, I82.B1x, I82.Clx, } \\
\text { K76.5, O22.3x, O22.5x, O87.1, O87.3, O88.2x, O88.8x, T82.81x, T82.86x, G08 }\end{array}$ \\
\hline \multirow[t]{2}{*}{ Bleedinga } & $\begin{array}{l}\text { ICD-9-CM: } 280,286.5,286.6,360.43,362.43,362.81,363.61,363.62,363.72,364.41,372.72,374.81,376.31,377.42,379.23, \\
\text { 380.31, 423, 430, 431, 432, 432.x, 455.5, 455.8, 456, 459, 530.7, 530.82, 531.0x, 531.2x, 531.4x, 531.6x, 532.0x, 532.2x, } \\
\text { 532.4x, 532.6x, 533.0x, 533.2x, 533.4x, 533.6x, 534.0x, 534.2x, 534.4x, 534.6x, 535._1, 537.83, 562._2, 562._3, 568.81, 569.3, } \\
\text { 569.85, 569.86, 578.x, 596.7x (except 599.72), 621.4, 623.6, 623.8, 624.5, 626.2, 626.6, 626.7, 626.8, 626.9, 719.1x, 782.7, } \\
\text { 784.7, 784.8, 786.3, 786.30, 864._1, 865._1, 866._1, 920, 921.x, 922.x, 923.x, 924.x, 958.2, 964.2, 997.02, 998.11, 998.12, } \\
\text { E858.2, E934.2 }\end{array}$ \\
\hline & $\begin{array}{l}\text { ICD-10: H05.23x, H21.0x, H31.30x, H31.41x, H35.6x, H35.73x, H43.10, H43.1x, H44.81x, H47.02x, H61.12x, I31.2, I60.x, } \\
\text { I61.x, I62.x, I85.01, K22.11, K25.0, K25.2, K25.4, K25.6, K26.0, K26.2, K26.4, K26.6, K27.0, K27.2, K27.4, K27.6, K28.0, } \\
\text { K28.2, K28.4, K28.6, K29._l, K31.81, K50._11, K51._11, K55.21, K57.01, K57._1, K57._3, K62.5, K66.1, K92.0, K92.1, K92.2, } \\
\text { M25.0x, M79.81, N42.1, N83.7, N89.7, N92.0, N92.1, N92.5, N93.0, N93.8, N93.9, O71.7, R04.x (except R04.81), R31.0, R31.9, } \\
\text { R58, S06.34_A, S06.35_A, S06.36_A, S06.37x, S06.38x, S06.4Xx, S06.5Xx, S06.6Xx, T79.2XXD, T79.2XXS }\end{array}$ \\
\hline
\end{tabular}

a Outcomes were not defined by ICD-9/10-CM codes. Rather, the diagnosis codes provided were used to identify all potential events that were then manually chart reviewed to validate all outcomes.

ICD-9/10-CM=International Classification of Diseases, Ninth/Tenth Revision, Clinical Modification. 


\section{APPENDIX B Baseline Characteristics Diagnosis Code Definitions}

Characteristic

History of hemorrhagic stroke

History of gastrointestinal bleed

History of other bleeding (nonhemorrhagic or gastrointestinal)

Heart failure

Diabetes mellitus

Hypertension

Coronary artery disease with a history of myocardial infarction

Coronary artery disease without a history of myocardial infarction

History of ischemic stroke

History of transient ischemic attack

History of peripheral arterial embolism

Renal dysfunction

Liver dysfunction

Peripheral arterial disease

Aortic plaque

History of alcohol abuse

\section{Definition}

ICD-9-CM: 430.x, 431.x, 432.x

ICD-10-CM: I60.x, I61.x, I62.x

ICD-9-CM: 531.0x, 531.2x, 531.4x, 531.6x, 532.0x, 532.2x, 532.4x, 532.6x, 533.0x, 533.2x, 533.4x, 533.6x, 534.0x, 534.2x, 534.4x, 534.6x, 535._1, 568.81, 578.x

ICD-10-CM: K22.11, K25.0x, K25.2x, K25.4x, K25.6x, K26.0x, K26.2x, K26.4x, K26.6x, K27.0, K27.2x, K27.4x, K27.6x, K28.0x, K28.2x, K28.4x, K28.6x, K29._1, K62.5x, K66.1x, K92.0x, K92.1x, K92.2x, D62.x

ICD-9-CM: 372.72, 362.81, 379.23, 627.0, 627.1, 784.7, 784.7, 784.8x, 599.7x, 459.0x

ICD-10-CM: J94.2, H11.3x, H35.6x, H43.1x, N02.x, N95.x, R04.x, R31.x, R58.x

ICD-9-CM: $428 \times$

ICD-10-CM: $150 . x$

ICD-9-CM: $250 . x$

ICD-10-CM: E10.x, 11.x

ICD-9-CM: 401.x, 402.x, 403.x, 404.x, 405.x

ICD-10-CM: I10.x, I11.x, I12.x, I13.x, I14.x, I15.x

ICD-9-CM: 410.x, 412.x

ICD-10-CM: I21.x, I22.x, I23.x, I25.2

ICD-9-CM: 411.x, 413.x, 414.x

ICD-10-CM: I20.x, I24.x, I25.x

ICD-9-CM: 433.x, 434.x, 436.x, 437.x, 438.x

ICD-10-CM: I63.x, I64.x, I65.x, I66.x, I67.x, I68.x, I69.x

ICD-9-CM: 435.x

ICD-10-CM: G45.x

ICD-9-CM: 444.x

ICD-10-CM: $174 . x$

ICD-9-CM: 582.x, 583.0x, 583.1x, 583.2x, 583.4x, 583.6x, 583.7x, 585.x, 586.x, 588.x

ICD-10-CM: I12.0x, I13.1x, N03.2x, N03.3x, N03.4x, N03.5x, N03.6x, N03.7x, N05.2x, N05.3x, N05.4x, N05.5x, N05.6x, N05.7x, N18.x, N19.x, N25.0x, Z49.x, Z94.0x, Z99.2x

ICD-9-CM: 070.32, 070.54, 456.1, 456.2x, 571.x, 572.x, V42.7

ICD-10-CM: B18.x, I85.x, I86.4x, I98.2x, K70.x, K71.1x, K71.3x, K71.4x, K71.5x, K71.7x, K72.x, K74.x, K76.0x, K76.2x, K76.3x, K76.4x, K76.5x, K76.7x, K76.8x, K76.9x, Z94.4x

ICD-9-CM: 440.2x, 440.3x, 440.4x, 443.81, 443.89, 443.9

ICD-10-CM: $170.2 \mathrm{x}, 170.26,170.29$

ICD-9-CM: 440.0

ICD-10-CM: 170.0

ICD-9-CM: 291.x (except 291.4), 303.9x, 305.0

ICD-10-CM: F10.x, E52.x, G62.1x, I42.6x, K29.2x, K70.0x, K70.3x, K70.9x, T51.x, Z50.2x, Z71.4x, Z72.1x 